\title{
Number of Previous Pregnancies
}

National Cancer Institute

\section{Source}

National Cancer Institute. Number of Previous Pregnancies. NCI Thesaurus. Code C120836.

A measurement of the total number of pregnancy events experienced by the female subject prior to the current pregnancy. 